\title{
Starspots Magnetic field by transit mapping
}

\author{
Adriana Válio ${ }^{1}$ and Eduardo Spagiari ${ }^{2}$ \\ ${ }^{1}$ CRAAM, Mackenzie University, São Paulo, Brazil, email: avalio@craam.mackenzie.brl \\ ${ }^{2}$ PGEE, Mackenzie University, São Paulo, Brazil, email: eduardo@spagiari.net
}

\begin{abstract}
Sunspots are important signatures of the global solar magnetic field cycle. It is believed that other stars also present these same phenomena. However, today it is not possible to observe directly star spots due to their very small sizes. The method applied here studies star spots by detecting small variations in the stellar light curve during a planetary transit. When the planet passes in front of its host star, there is a chance of it occulting, at least partially, a spot. This allows the determination of the spots physical characteristics, such as size, temperature, and location on the stellar surface. In the case of the Sun, there exists a relation between the magnetic field and the spot temperature. We estimate the magnetic field component along the line-of-sight and the intensity of sunspots using data from the MDI instrument on board of the SOHO satellite. Assuming that the same relation applies to other stars, we estimate spots magnetic fields of CoRoT-2 and Kepler-17 stars.
\end{abstract}

Keywords. Sunspots, star spots, stellar magnetic field

\section{Sunspots}

Dark spots observed on the surface of the Sun are important signatures of its global magnetic field. It is believed that other stars also behave the same way. However, currently it is not possible to directly observe spots on the surface of other stars due to their small sizes, which are a fraction of an arc sec. The method proposed by Silva (2003) to study spots on other stars is based on the small variations detected during planetary transits. During a planetary transit, as the planet passes in front of its parent star, there is the possibility it will occult, at least partially, a region darker or brighter than the stellar surface. A detailed analysis of the star light curve may show small variations caused by these occultations. Since the spot is darker than the rest of the stellar disk, an increase in the light intensity during the transit is seen. The fit to these light curve variations yields physical characteristics of the spots, such as the size, temperature and location on the surface of the star. Therefore, these planets can be used as probes to study the characteristics of stellar surface features such as spots. To estimate the starspots magnetic field, we assume that starspots follow the same relation between intensity and magnetic field known for sunspots.

The first step is to obtain an empirical relation between the intensity of sunspots and their magnetic field. The solar data used here were obtained from the MDI instrument on board of the SOHO (Solar and Heliospheric Observatory) satellite. A total of 226 spots were identified within the solar images for the whole year of 2003. Their area and average intensity with respect to the central disk intensity were determined as in Spagiari et al. (2012) from the white light images. The magnetic field was estimated from the corresponding magnetograms, both the maximum and minimum magnetic intensity were recorded within the same area used in the white light images. For better precision of the data, and to avoid projection effects, only spots located between longitudes $-40^{\circ}$ and $40^{\circ}$ were analyzed, totaling 137 sunspots.

Next, the spot relative intensity is converted to temperature by assuming that both the photosphere and the spots radiate like a blackbody. Thus, the spot temperature is 

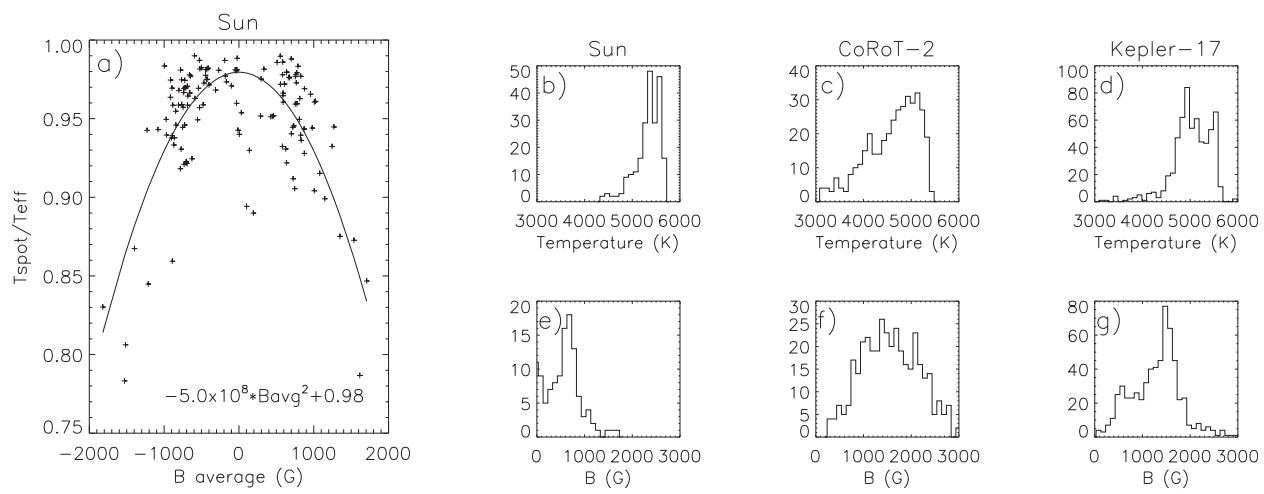

Figure 1. a) Sunspots temperature versus average magnetic field intensity. Temperature of spots on the Sun (b), CoRoT-2 (c), and Kepler-17(d). Maximum magnetic fields of sunspots (e), and spots on CoRoT-2 (f) and Kepler-17 (g) stars.

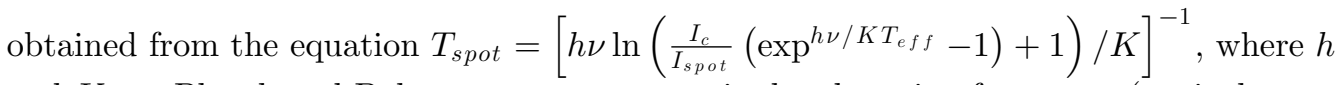
and $K$ are Planck and Boltzmann constants, $\nu$ is the observing frequency (equivalent to $\lambda=600 \mathrm{~nm}), T_{\text {eff }}$ is the solar effective temperature $\left(T_{\text {eff }}=5780 \mathrm{~K}\right), I_{\text {spot }}$ is the spot intensity and $I_{c}$ the maximum intensity of the Sun at disk center.

\section{Spots Results and Conclusions}

Figure 1a shows the values of sunspots temperature (Figure 1b) as a function of their average magnetic field intensities (Figure 1e). From observation of sunspots, Dicke (1970) obtained the empirical relation: $T_{\text {spot }} / T_{\text {eff }}=-3.21 \times 10^{-8} B^{2}+0.95$, or $T_{\text {spot }} / T_{\text {eff }}=$ $\alpha B^{2}+\sigma$. Following Dike (1970), quadratic fit of the sunspot data are shown for spots located between longitudes $\pm 40^{\circ}$ as a solid curve on Figure 1a. The estimated values from the fit on Figure 1a are $\alpha=(-5.0 \pm 0.4) \times 10^{-8} G^{-2}$ and $\sigma=0.980 \pm 0.003$.

The next step is to use this empirical relation to estimate magnetic fields of stellar spots. Two stars with transiting planets were analyzed: CoRoT-2 $\left(T_{\text {eff }}=5575 \mathrm{~K}\right)$ and Kepler-17 $\left(T_{e f f}=5781 \mathrm{~K}\right)$. Small variations in the transit light curves of these stars have been fitted and a total of 392 spots for CoRoT-2 (Silva-Valio \& Lanza 2011) and 615 spots on Kepler-17 where characterized and their areas and intensities obtained. These spot intensities where then converted to temperatures (Figures $1 \mathrm{c}$ and $\mathrm{d}$ for the stars CoRoT-2 and Kepler-17, respectively).

Assuming that the decrease in intensity, or temperature, is caused by more intense magnetic fields, and that the spot intensity-magnetic field relation follows that of the Sun, the empirical relation defined above can be inverted to yield the magnetic field for a given relative temperature. Histograms of the derived maximum magnetic field for the stars CoRoT-2 and Kepler-17 are shown in Figures 1f and g, respectively.

The average fields obtained this way for sunspots are $700 \pm 350 \mathrm{G}$, whereas the spots on the stars CoRoT-2 and Kepler-17 have average magnetic field intensities of $1700 \pm 700$ $\mathrm{G}$ and $1400+500 \mathrm{G}$, respectively. As can be seen from the results, the youngest star CoRoT-2, that is also the most active, has the most intense magnetic fields.

\section{References}

Dicke, R. H. 1970, ApJ, 159, 25

Silva, A. V. R. 2003, ApJ, 585, L147

Silva-Valio, A. \& Lanza, A. F. 2011, A\&A, 529, 36

Spagiari, A. E., Santos, I. F., Costa, W. L. , Valio, A., \& Marengoni, M. 2012, in: Avancos em Visao Computacional (Omnipax Editor), pp. 345-364. 